\title{
MODELAGEM CLIMÁTICA REGIONAL DURANTE DOIS ANOS DE EXTREMOS DE PRECIPITAÇÃO SOBRE O ESTADO DO AMAPÁ: TESTE DE SENSIBILIDADE AOS ESQUEMAS CONVECTIVOS
}

\author{
DANIEL GONÇALVES DAS NEVES ${ }^{1,2}$, ALAN CAVALCANTI DA CUNHA ${ }^{1}$, EVERALDO BARREIROS \\ DE SOUZA ${ }^{3}$, NAURINETE JESUS DA COSTA BARRETO ${ }^{4}$
}

\author{
${ }^{1}$ Universidade Federal do Amapá, Programa de Pós-Graduação em Biodiversidade Tropical (UNIFAP/ \\ PPGBIO), Macapá, AP, Brasil \\ ${ }^{2}$ Instituto de Pesquisas Científicas e Tecnológicas do Estado do Amapá, Núcleo de Hidrometeorologia e \\ Energia Renováveis (IEPA/NHMET), Macapá, AP, Brasil \\ ${ }^{3}$ Universidade Federal do Pará, Faculdade de Meteorologia (UFPA/FAMET), Belém, PA-Brasil \\ ${ }^{4}$ Universidade Federal do Rio Grande do Norte (UFRN), Natal, RN-Brasil
}

daniel.neves@iepa.ap.gov.br, alancunha@unifap.br, everaldo@ufpa.br, netebarreto@gmail.com

Recebido Fevereiro 2010 - Aceito Julho de 2011

\begin{abstract}
RESUMO
O presente estudo mostra uma avaliação da previsão sazonal do Modelo RegCM3 em dois eventos extremos de precipitação, nos anos 2006 e 2007. Na análise foi utilizada a resolução horizontal de $1^{\circ} \mathrm{x} 1^{\circ} \mathrm{km}$ e validado com $30 \times 15$ pontos de latitude x longitude para a região do Estado do Amapá. Os resultados apresentados são referentes à comparação entre precipitação sazonal simulada e observada para o trimestre (MAM). De maneira geral, os resultados mostraram que a utilização da técnica de redução de escala de previsão da precipitação sazonal, apresentou um bom desempenho em simular a variabilidade da chuva em escala regional. Porém, erros na quantidade e na posição de alguns máximos foram observados, quando comparado com os dados observados. O viés úmido foi predominante na região litorânea e o viés seco nas áreas sul-sudeste, oeste e centro com forte persistência na região sudeste. Não se observou regiões com valores estimados de precipitação próximos aos valores observados.
\end{abstract}

Palavras-chave: Precipitação Sazonal, Variabilidade, Amapá, Modelagem Climática.

\begin{abstract}
REGIONAL CLIMATE MODELING FOR TWO YEARS OF EXTREME PRECIPITATION OVER THE STATE OF AMAPA: TEST OF SENSITIVITY TO CONVECTIVE SCHEMES

This study shows an assessment of the seasonal forecast model RegCM3 in two extreme events of precipitation for the years 2006 and 2007. For the analysis a $1^{\circ} \times 1^{\circ} \mathrm{km}$ horizontal resolution was used and validated with 30 x 15 points of latitude x longitude for the region of the State of Amapa. The presented results are for the comparison between simulated and observed seasonal precipitation in the quarter (MAM). In general, the results showed that using the scale reduction technique to predict the seasonal rainfall, a good performance in simulating the variability of rainfall on a regional scale is obtained. However, errors in the precipitation amount and on some maximum position were observed when compared with the observed data. The wet bias was prevalent in the humid coastal region and the dry bias in the south-southeast, west and center with a strong persistence in the Southeast. Regions with precipitation values close to the observed ones were not very evident.
\end{abstract}

Keywords: Seasonal Precipitation, Variability, Amapa, Climatic Modelling 


\section{INTRODUÇÃO}

A região Amazônica é conhecida pela exuberância de suas florestas tropicais e regime pluviométrico elevado, apresentando uma significativa variabilidade espacial e temporal do clima, que pode ser verificada pela variação na distribuição de precipitação sobre diversos locais da região e em diferentes épocas do ano (Figueroa e Nobre, 1990; Lopes, 2009). Tal variabilidade climática, em diversas escalas, tem sido motivo de preocupação de vários autores na tentativa de explicar coerentemente questões complexas, como por exemplo: como os ecossistemas podem ser impactados em um contexto de rápida mudança da dinâmica atmosférica?

Em um estudo recente observou-se um significativo ciclo diurno da atividade convectiva da Zona de Convergência Intertropical (ZCIT) em sua posição no Atlântico equatorial, sendo este sistema um dos principais mecanismos reguladores da variabilidade diurna de precipitação que se estende da costa do Pará até o Amapá (Souza e Rocha, 2006). Lopes (2009) acrescenta, ainda, que a ZCIT é um fenômeno meteorológico de escala intra-sazonal que influencia a distribuição de precipitação,e em muitos casos, é responsável pela variabilidade no clima regional.

Lopes (2009) descreve em seus estudos sobre o clima do Pará e Amapá que as condições oceânicas, principalmente a temperatura da superfície do mar (TSM), também apresentam marcante influência sobre o clima global e regional, cujas evidências regionais e simulações numéricas têm confirmado tais hipóteses. Exemplos disto são as conexões entre a variabilidade interanual da circulação de grande escala sobre o Atlântico Tropical e as alterações na pluviometria local.

Neste contexto, os estudos e pesquisas específicas do clima para o Estado do Amapá,em especial no que se refere ao comportamento da precipitação e seu clima atual, têm se mostrado insuficientes na geração de informações consistentes e atualizadas. Portanto, a principal contribuição de trabalhos nesta área é a de promover acréscimos no desenvolvimento da climatologia acoplada às necessidades da gestão, preservação e conservação da biodiversidade tropical local. Por outro lado, estudos de modelagem climática,cujo objetivo seja o de suprir a necessidade de conhecimento sobre a variabilidade de chuva no Estado do Amapá, têm se tornado a cada dia extremamente necessários. A razão são àsfrequentes ocorrências de eventos extremos no Estado.

O comportamento da precipitação sobre a Região Amazônica é um tema bastante investigado (Souzae Rocha,2006; Souza et al., 2009;Lopes, 2009; entre outros). Mas quando se trata do Estado do Amapá, há lacunas imensas a serem preenchidas com relação aoclima local. Logo, as razões que justificam tais estudos são aquelas em que o interesse crescente por dados e informações meteorológicas, no contexto global e regional, se apresenta como fator preponderante nas tomadas de decisões em praticamente todas as áreas do conhecimento humano. Bons exemplos são os diagnósticos e prognósticos do clima em situações de riscos de ocorrência de eventos extremos, que afetam cada vez mais as populações, em especial as mais vulneráveis ao clima, como as de baixa renda ou condição socioeconômica adversa.

Por outro lado, os modelos de circulação global (MCB) não são capazes de resolver satisfatoriamente os detalhes da fisiografia bem como a circulação local de certas regiões. Neste aspecto, há a vantagem do uso de modelos climáticos regionais (Lopes, 2009). Além disso, os modelos regionais são ferramentas que apresentam melhor resolução espacial, quando comparados aos modelos globais, o que permite verificar com mais detalhes os aspectos da interação superfície-atmosfera (Giorgi e Mearns, 1999).

Inúmeros estudos de modelagem climática regional sobre a América do Sul, Brasil e região Amazônica têm sido realizados para investigar o desempenho dos modelos regionais. Seth e Rojas (2003) investigaram a capacidade do RegCM2 em reproduzir a variabilidade interanual de precipitação e a circulação sobre a região, comparando simulações para anos de El Niño (1983) e La Niña (1985). Neste caso, o RegCM2 simulou corretamente os diferentes padrões da circulação em grande escala e as anomalias nestes padrões, e precipitação associada.

Alves et al.(2004) analisaram previsões sazonais através do ETA climático para o ano de 2003 sobre o Brasil. Os referidos mostraram resultados em que os totais pluviométricos gerados pelo modelo regional, no verão, foram subestimados e, no inverno, foram superestimados. Porém previu consideravelmente melhor a variabilidade intrazasonal quando comparado com o modelo global.

Fernandez et al. (2006) investigaram as circulações quase-estacionárias (Alta da Bolívia) e os efeitos das anomalias de grande escala (El Niño 97/98 e La Niña 98/99), utilizando dois modelos regionais (ETA CLIM e RegCM3) para uma simulação de 10 anos. Ambos os modelos tiveram êxito em simular com coerência a variabilidade interanual durante os eventos extremos. Cuadra e Rocha (2006) realizaram simulações com o RegCM3 e investigaram a variabilidade de precipitação, variabilidade de temperatura do ar e circulações em baixos níveis, durante o verão de 1990 a 1998 para o sudeste do Brasil. Resultados mostraram que os padrões de variabilidade de precipitação, de temperatura e anomalia de circulação são consistentes com os dados de re-análise do National Centers for Environmetal Prediction/National Center for Atmospheric Research (NCEP/NCAR).

Souza et al. (2009), em um estudo recente de modelagem climática para a região da Amazônia oriental,utilizando 
simulações sazonais e o modelo regional RegCM3 para um período de 26 anos, com resolução de $30 \mathrm{~km}$, observou que o RegCM3 simulou com mais eficácia os padrões anômalos de precipitação regional usando a parametrização de Grell, e apresentou melhor desempenho da precipitação simulada na região da Zona de Convergência do Atlântico Sul (ZCAS), quando comparado com as simulações para a região da Zona de Convergência Intertropical (ZCIT).

Assim, o objetivo deste trabalho é avaliar o desempenho do modelo RegCM3 em simular a precipitação sazonal em dois anos (2006 e 2007) de ocorrência de precipitação extrema sobre o Estado do Amapá.

\section{DADOS E METODOLOGIA}

\subsection{Dados de precipitação}

A ausência de uma maior cobertura espacial de estações meteorológica, distribuída em toda região norte do Brasil,impede um melhor monitoramento de eventos extremos que ocorrem na região. Os dados usados no estudo observacional e na comparação com os resultados da modelagem numérica tiveram como base os dados precipitação diária do Climate Prediction Center (CPC), para uma grade sobre o Brasil com resolução espacial $1^{\circ}$ x $1^{\circ}$ latitude por longitude. Estes dados foram recentemente avaliados para simulações de modelagem climática, e podem ser usados como base para monitoramento do tempo e ocorrência de eventos extremos (Silva et al., 2007).

\subsection{Processo de redução de escala (downscaling dinâmico)}

Atualmente, diferentes técnicas de "downscaling" têm sido desenvolvidas baseadas em métodos dinâmicos ou estatísticos, buscando adicionar características regionais do clima, como topografia e cobertura do solo, aos resultados dos modelos climáticos globais (Quian et al., 2003; Frias et al., 2005).

O método usadono presente trabalho é a técnica de downscaling dinâmico, o qual consiste no uso de um modelo climático regional (RegCM3) com alta resolução horizontal, usando como condição de contorno o resultado das simulações dos modelos climáticos globais. Desta maneira o modelo climático regional (RegCM3)pode produzir circulações ausentes nos modelos climáticos globais (Druyan et al., 2002). Portanto, com a utilização do modelo regional RegCM3, é esperada uma melhora nas simulações climáticas para o Estado do Amapá, com ênfase na variável de precipitação que apresenta forte variabilidade sobre o Estado (Souza et al., 2009; Lopes., 2009).

\subsection{Descrição do $\operatorname{RegCM3}$}

O Regional Climate Model 3 (RegCM3) é a última versão desenvolvida pelo International Center for Theoretical Physics (ICTP) (Pal et al., 2007). O RegCM3 é a terceira geração de modelagem climática regional originalmente desenvolvida no NCAR (Dickinsonet al.,1993). O modelo é uma versão melhorada da versão 2.5 (RegCM2.5) descrito por Giorgi e Mearns (1991). Estas melhoras consistiram na representação física da precipitação, superfície continental, química da atmosfera e aerossóis. Mudanças importantes no desempenho do modelo têm sido realizadas, no pré-processamento, rodada e pós-processamento do RegCM3 (Pal et al., 2007).

Basicamente o ITCP RegCM3 é um modelo de área limitada, em coordenada vertical sigma e grade horizontal de Arakawa-Lamb B, com as equações primitivas para fluido compressível e hidrostático.

O RegCM3 usa o esquema de superfície BiosphereAtmosphere Transfer Scheme (Dickinson et al., 1993) para incorporar os processos de superfície, considerando a presença de vegetação em cada ponto de grade e sua interação com as camadas de solo nas trocas turbulentas de momentum,energia e vapor d'água entre a superfície e a atmosfera. O transporte turbulento dessas quantidades resulta do produto entre o respectivo gradiente vertical e o coeficiente de difusão vertical turbulenta, segundo as correções para turbulência não local proposta por Holtslag et al. (1990).

Para transferência Radiativa o modelo utiliza o mesmo esquema da Community ClimateModel 3 (Kiehlet al., 1996). Neste esquema, as taxas de aquecimento e fluxos na superfície para radiação solar e infravermelha sob condições de céu claro e nublado, são calculados separadamente. Os cálculos de transferência radiativa consideram os efeitos dos gases $\mathrm{CO}_{2}$ e $\mathrm{O}_{3}$ no infravermelho e vapor de $\mathrm{H}_{2} \mathrm{O}$, bem como dos gases $\mathrm{CO}_{2}, \mathrm{O}_{3}$ e $\mathrm{O}_{2}$ e vapor de $\mathrm{H}_{2} \mathrm{O}$ para radiação solar. $\mathrm{O}$ esquema inclui também os efeitos dos gases de efeito estufa $\left(\mathrm{NO}_{2}, \mathrm{CH}_{4}\right.$, CFCs), aerossóis atmosféricos e água de nuvem.

No presente trabalho foram utilizadosdois tipos de parametrização convectiva presentes no modelo. A primeira parametrização utilizada foi a de Grell (Grell, 1993), em que a convecção de cumulus é representada por correntes ascendentes e descendentes que só se misturam com o ar ambiente na base e no topo das correntes. Os perfis verticais de aquecimento e umedecimento são derivados da liberação de calor latente associada com os fluxos de massa nas correntes ascendentes/ descendentes e movimento vertical de compensação. Neste trabalho, foi utilizado o fechamento de Fritsch-Chapell (Elguindi et al., 2004), o qual considera que a energia de flutuação disponível ocorre numa escala de tempo de 30 minutos. A segunda parametrização é a de MIT-Emanuel (Emanuel, 1991), a qual 
considera que a mistura das nuvens é um fenômeno altamente não homogêneo que consiste de fluxos convectivos baseados em um modelo ideal de correntes descendentes e ascendentes na escala de sub-nuvem. A convecção é provocada quando o nível de neutralidade é maior do que o nível da base da nuvem. Entre esses dois níveis, o ar é levantado e uma fração da umidade condensada é convertida em precipitação enquanto a fração restante é convertida em nuvem (Souzaet al., 2009; Lopes, 2009).

Para representar a precipitação em grande escala (Pal et al., 2007) foi aplicado o esquema SUB-grid-EXplicit moisture scheme (SUBEX). O SUBEX considera formação de água a nuvem, advecção e mistura turbulenta, re-evaporação em condiçõessub-saturadas, "acreção" e conversão para precipitação através de um termo de auto-conversão.

Para esta pesquisa, a dimensão da grade do RegCM3 configurada para a região do Estado do Amapá foi de 30 x 15 pontos de latitude e longitude, com resolução horizontal de $1^{\circ} \mathrm{x}$ $1^{\circ}(\sim 111 \mathrm{Km})$, centrado em $\left(1.5^{\circ} \mathrm{N}: 52.0^{\circ} \mathrm{W}\right)$ (Figura 1$)$ com 18 níveis na vertical (sendo 7 níveis abaixo de $800 \mathrm{hPa}$ ) e usando projeção mercator normal. Foram realizadas duas simulações sazonais usando os esquemas de convecção de Grell e MITEmanuelpara o período de janeiro a junho, sendo que uma vez que o foco deste trabalho é sobre o período chuvoso da Amazônia oriental, as simulações foram iniciadas em 01 de janeiro e finalizadas em 30 de junho, considerando o tempo de "spin-up" o primeiro mês da integração. As rodadas foram atualizadas (updated) a cada 6 horas com as condições iniciais e de contorno da reanálise NCEP/NCAR (Kalnay et al., 1996) e usando os dados mensais observados de TSM de Reynolds et al. (2002).

\subsection{Técnica estatística utilizada no estudo}

O método usado para selecionar os anos extremos de precipitação foi a "técnica dos quantis", pois procura interpretar de forma adequada o verdadeiro significado de um total pluviométrico. Na Tabela 1 são apresentados os intervalos de classes para a categorização da precipitação conforme o critério de Xavier e Xavier (1987). Neste estudo foram convertidos em quantis os dados pluviométricos da estação de Macapá provenientes do Instituto Nacional de Meteorologia (INMET), no período entre 1978e 2008. Segundo esta metodologia, os anos selecionados a partir da técnica utilizada foram 2006 (muito seco) e 2007 (muito chuvoso), onde foi destacado e analisado apenas o trimestre (março-abril-maio) mais chuvoso para o Estado do Amapá.

\subsection{Parâmetro de avaliação do modelo RegCM3}

Para quantificar o desempenho da modelagem dinâmica de simulação sazonal para o trimestre março-abril-maio (MAM) mais chuvoso dos anos 2006 e 2007 do Estado do Amapá, foi adotado o viés, que é uma medida do erro sistemático do método, dado pela formulação abaixo.

$$
B_{i j}=\frac{1}{n}\left(P_{i j}-O_{i j}\right)
$$

onde $n$ é o número de anos de avaliação, os índices $i$ e $j$ indicam as coordenadas dos pontos de grade, e P e O são os valores previstos e observados.

Tabela 1- Intervalos de classes para categorização da precipitação.

\begin{tabular}{lcc}
\hline \hline MUITO SECO & quando & $\mathrm{xi} \leq \mathrm{Q}(0,15)$ \\
\hline SECO & quando & $\mathrm{Q}(0,15)<\mathrm{xi} \leq \mathrm{Q}(0,35)$ \\
\hline NORMAL & quando & $\mathrm{Q}(0,35)<\mathrm{xi}<\mathrm{Q}(0,65)$ \\
\hline CHUVOSO & quando & $\mathrm{Q}(0,65) \leq \mathrm{xi}<\mathrm{Q}(0,85)$ \\
\hline MUITO CHUVOSO & quando & $\mathrm{xi} \geq \mathrm{Q}(0,85)$ \\
\hline \hline
\end{tabular}

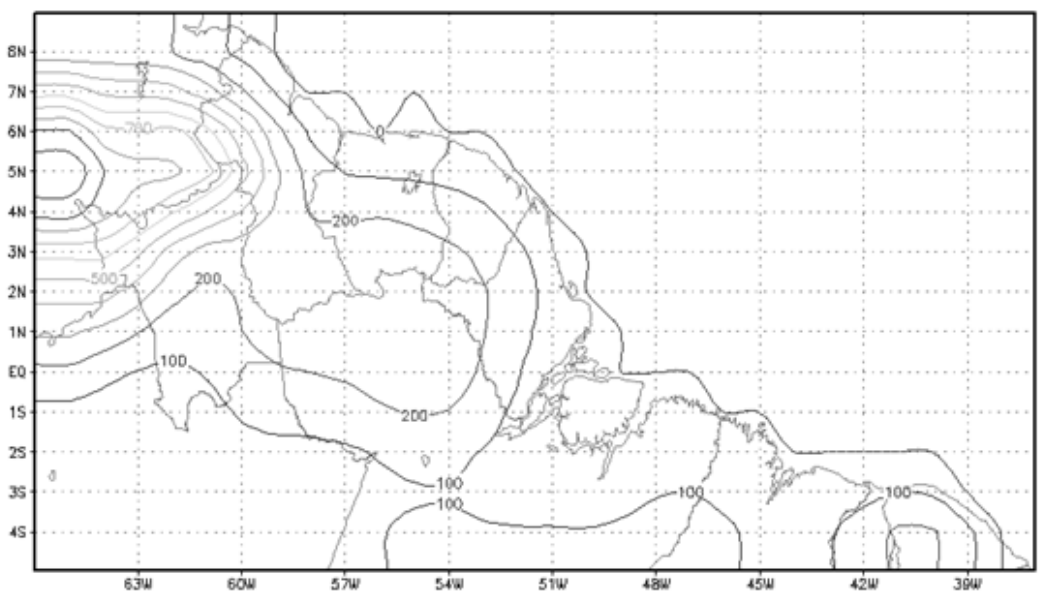

Figura 1 - Domínio do modelo RegCM3 com resolução horizontal de $1^{\circ}$ x $1^{\circ}(\sim 111 \mathrm{Km})$. As isolinhas indicam a topografia em metros. 


\section{RESULTADOS E DISCUSSÃO}

\subsection{Seleção dos anos extremos de precipitação}

No presente trabalho foi convertido em quantis os dados pluviométrico da estação de Macapá-AP. As medidas trimestrais de precipitação foram normalizadas entre 2 e -2 para uma série histórica de 1978 a 2008. Na Tabela 2 é indicado como a técnica dos quantis classifica os $15 \%$ dos trimestres dos anos como muito seco, $20 \%$ seco, $30 \%$ normal, $20 \%$ chuvoso e $15 \%$ muito chuvosos.

\subsection{RegCM3 x CPC: Análise qualitativa}

Na Figura 2verifica-se os campos de precipitação estimados do CPC e simulados pelo RegCM3, utilizando as parametrizações de Grell e MIT-Emanuel para os meses de março-abril-maio(MAM) do ano de 2006. Em uma análise qualitativa dos resultados, verificou-se que a simulação, utilizando o esquema convectivo de Grell (Figura 2a), para o mês de março, superestima os dados de precipitação ao norte, centrooeste, extremo leste e sul do Estado do Amapá, com núcleo de precipitação de $700 \mathrm{~mm}$ situado na região central do Estado, e subestima nas regiões oeste, sudeste e parte do sul do Amapá. No mês de abril (Figura $2 b$ ), notou-se uma superestimativa ao norte e extremo oeste e subestimativa no sudeste, oeste-sudoeste e a parte da região central. Já para o mês de maio (Figura 2c), ocorreu uma superestimativa de precipitação em praticamente todo o Estado, com um núcleo de subestimativa $(100 \mathrm{~mm})$ sobre o município de Macapá. No esquema de MIT para oreferido trimestre (MAM) de 2006, foi verificado que a precipitação simulada foi muito próxima da observada, nos meses de março e abril, com exceção do mês de maio que apresentou uma superestimativa em quase todo o Estado.

Nos dois esquemas de parametrização simulados foi observado um núcleo de subestimativa de precipitação localizado principalmente sobre capital do Estado. Os resultados da subestimativa de precipitação nas simulações do RegCM3, foram consistentes com estudos anteriores de simulações para a Amazônia oriental(Souza et al., 2009)e Brasil (Alves et al., 2004).Já em relação ao mês de maio, ocorreu um predomínio de superestimativa em quase todo o Estado, para a simulação tanto de Grell como MIT. Isto, pode se dever ao fato, que este é um mês de transição do período chuvoso para o menos chuvoso na Amazônia oriental (Amapá). Ou seja, as chuvas começam a reduzir, tendo como característica, uma chuva mais fraca e contínua ao longo do dia (Souza e Rocha, 2006), resultando numa simulação do RegCM3 de valores elevados em relação ao observado.

No ano de 2007 (Figura 3a) foi observado para o esquema convectivo de Grell, que ocorreu uma superestimativa na precipitação, com destaque para a região litorânea do Estado. Já nos meses de abril e maio (Figura 3b,c), esta superestimativa ficou distribuída em praticamente toda a região do Estado. Com o esquema de MIT-Emanuel, simulado para o mês de março (Figura 3a), os resultados apresentaram valores muito próximos dos observados, e os meses de abril e maio (Figura $3 \mathrm{~b}, \mathrm{c}$ ) apresentaram novamente uma superestimativa em boa parte do Amapá. Para o trimestre estudado nos dois anos, foi observado nas simulações com Grell e MIT-Emanuel, um núcleo de subestimativa de chuva localizado na região sudeste da região de estudo, concordando com os resultados de Alves et al. (2004)e Souza et al. (2009).

Logo, nesta primeira análise foi observado que o modelo RegCM3 conseguiu capturar a variabilidade da chuva no trimestre dos dois anos estudados, apresentando erros na quantidade e no posicionamento dos máximos e mínimos de chuva sobre o Estado. Como esta análise foi feita no final da estação chuvosa no Estado, foi observada uma retração dos máximos pluviométricos simulados pelo modelo no esquema de Grell,com destaque para o ano de 2006 (Figura 2b), que por sua vez está associada com o deslocamento meridional da ZCIT para o Atlântico Norte, conforme mostrado por Souza et al. (2004).

Tabela 2 - Classificação dos anos (1978-2008) usando a técnica dos quantis.

\begin{tabular}{ccccc}
\hline \hline Muito seco & Seco & Normal & Chuvoso & Muito chuvoso \\
\hline \hline 1981 & 1980 & 1979 & 1982 & 1978 \\
1983 & 1987 & 1989 & 1984 & 1985 \\
2003 & 1993 & 1990 & 1988 & 1986 \\
2006 & 1997 & 1991 & 1996 & 1994 \\
& 1998 & 1992 & 2000 & 2007 \\
& 2005 & 1995 & 2002 & \\
& & 1999 & & \\
& & 2004 & & \\
& & 2008 & & \\
& & & & \\
\hline \hline
\end{tabular}




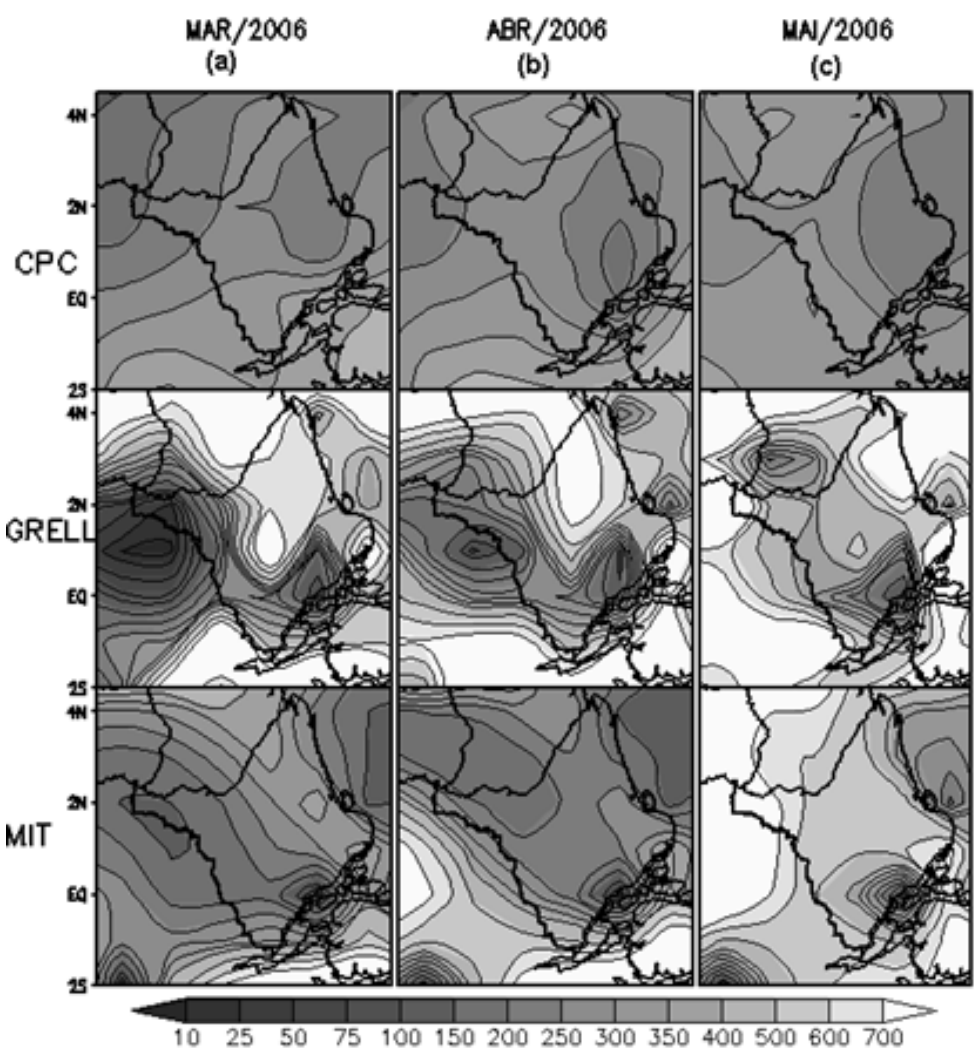

Figura 2 - Dados de precipitação observada pelo CPC e simulada utilizando o esquema de Grell e MIT sobre o Estado do Amapá para o ano de 2006. (a) Março, (b) Abril, (c) Maio. A escala de cores abaixo da figura indica a intensidade em mm/mês.

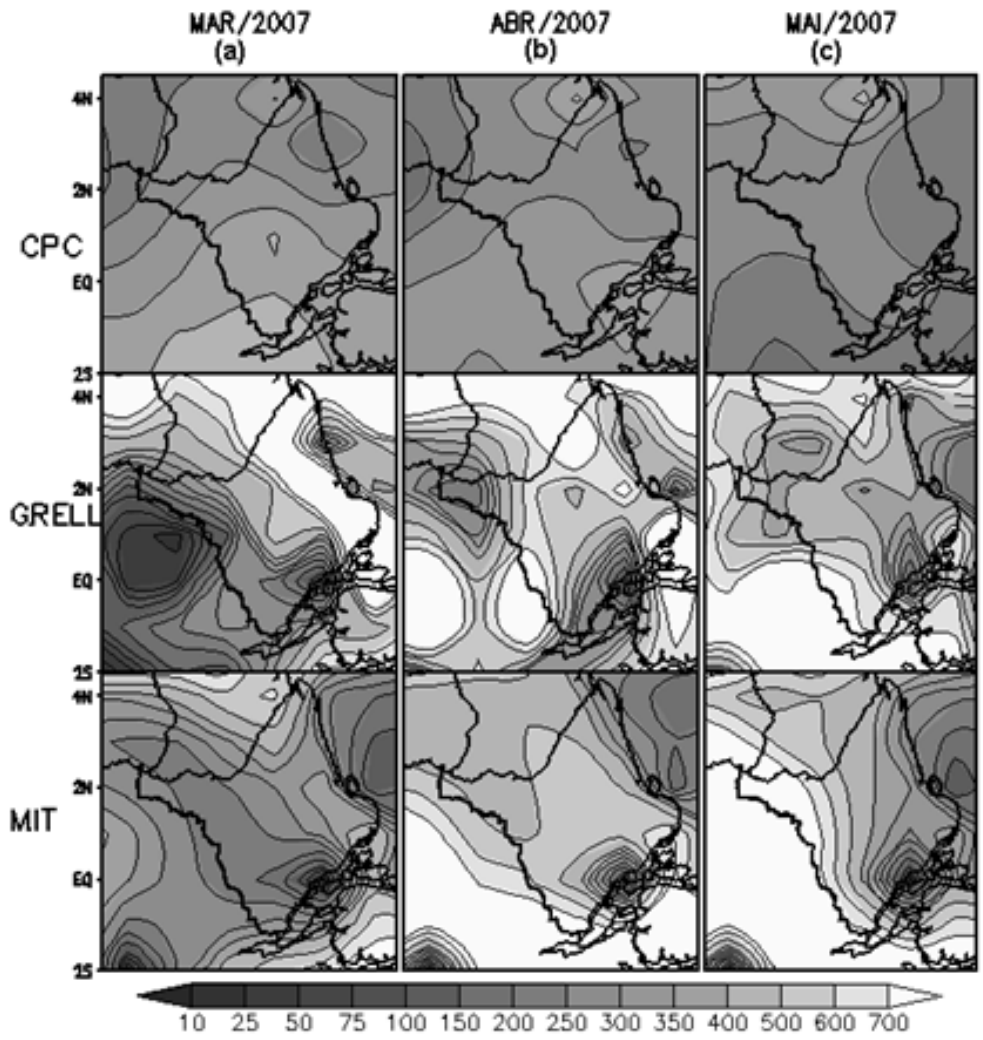

Figura 3 - Dados de precipitação observada pelo CPC e simulada utilizando o esquema de Grell e MIT sobre o Estado do Amapá para o ano de 2007. (a) Março, (b) Abril, (c) Maio. A escala de cores abaixo da figura indica a intensidade em mm/mês. 


\subsection{RegCM3 x CPC: Análise quantitativa}

Para uma análise quantitativa (intensidade da diferença entre o simulado e o observado),os resultadossão interpretados como viés seco (isolinha negativa) e viés úmido (isolinha positiva). As diferenças próximas de zero indicam que a simulação está mais próxima do valor observado.

Na Figura 4a para o ano de 2006 (muito seco) utilizando a parametrização de Grell, foi observado o viés seco numa faixa que vai desde as regiões sudeste e sudoeste chegando até o oeste do Estado do Amapá,nos meses de março e abril. Já para o mês de maio o viés seco foi evidenciado apenas na região sul-sudeste com intensidade de $-150 \mathrm{~mm}$ sobre o Município de Santana e Macapá. O viés úmido apresentou certo predomínio em todo o trimestre (MAM) estudado, com diferenças quantitativas maiores localizadas nas regiões central-norte, litoral e extremo leste do Estado. Na parametrização de MIT(Figura 4b) foi observado um comportamento oposto ao de Grell. Ou seja, ocorreu maior predomínio do viés seco em quase toda a área do Estado, com exceção do mês de maio, cujo viés úmido foi predominante, com uma pequena área de viés seco localizada sobre a cidade de Macapá.

As regiões com valores próximo do observado (valores próximo de zero) não apresentaram grandes extensões nos resultados, com destaque na porção sul-sudeste do Amapá, indicadas nas Figuras $4 \mathrm{a}$ e $4 \mathrm{~b}$ das análises.

$\mathrm{Na}$ Figura 5a do ano de 2007 (muito chuvoso), para o mês de março, foi verificado o viés úmido nas regiões norte, centro-norte e leste-nordeste, com intensidade de $400 \mathrm{~mm} / \mathrm{mês}$, se estendendo em toda a faixa litorânea do Amapá. Nas áreas oeste, sul-sudoeste e parte do sudeste, foi registrado o viés seco, com intensidade (-400 mm/mês) abrangendo o município de Macapá e Santana. Para os meses de abril e maio foi observado o predomínio do viés úmido em grande parte do Estado, com apenas uma pequena área de viés seco persistindo sobre os municípios de Macapá e Santana.

$\mathrm{Na}$ Figura 5b, para a simulação usando o esquema de MIT, diferente da simulação de Grell, foi verificado que o mês de março apresentou uma amplificação de viés seco em grande parte do Estado,com maior intensidade $(-400 \mathrm{~mm} / \mathrm{mês})$ sobre Macapá e Santana. O viés úmido ficou novamente distribuído em toda a faixa litorânea. No mês de abril e maio os resultados apresentaram características muito semelhantes, com viés úmido em praticamente todo o Estado e viés seco sobre a região sudeste (Macapá e Santana).

Nas duas análises das Figuras 5a e 5b, foram verificadas pequenas faixas de valores que se aproximam do observado em diferentes áreas do trimestre estudado, com destaque naparametrização de Grell do mês de abril que apresentou valores próximos do observado, com maiores áreas situadas no extremo oeste do Amapá.

De maneira geral o RegCM3 apresentou um predomínio de viés úmido em todo trimestre estudado para os dois anos extremos de precipitação. Estes resultados estão de acordo com Alves etal. (2007), que encontraram um viés úmido para a região do nordeste do Brasil e parte da região Amazônica, utilizando um Modelo Regional Espectral (MRE/97) para o período de fevereiro-março-abril (FMA) e março-abril-maio (MAM) e contrapondo-se a Alves et al. (2004), que encontraram resultados subestimados de precipitação para o Estado do Amapá.

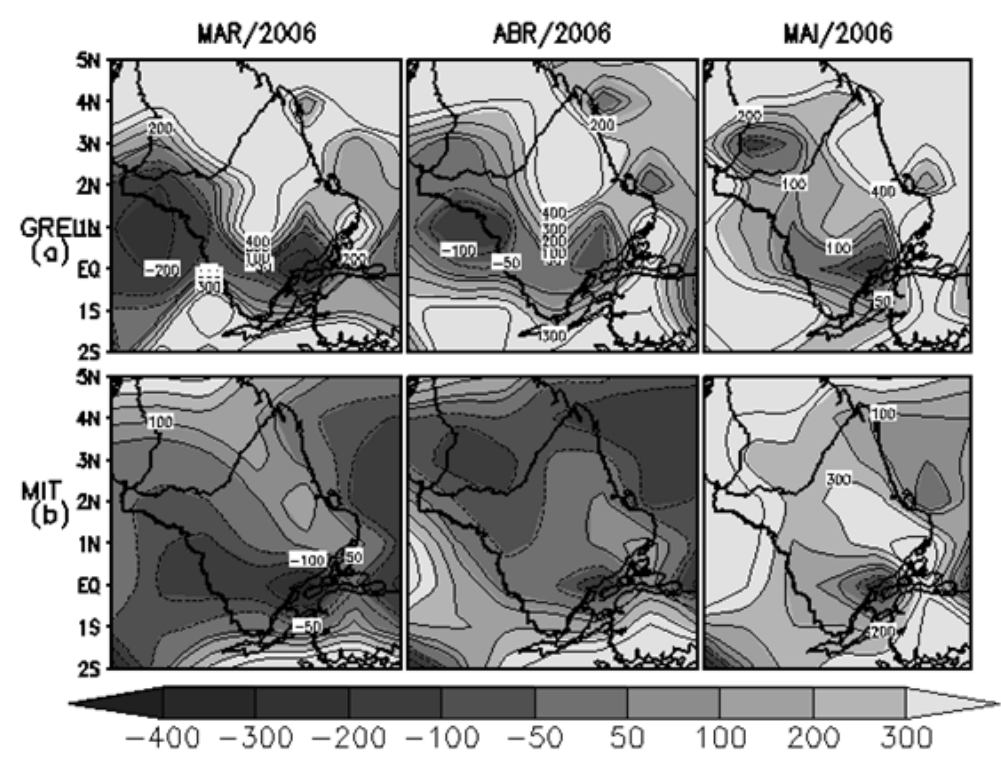

Figura 4 - (a) Viés usando o esquema de Grell, (b) Viés usando o esquema de MIT para os meses de março, abril e maio do ano de 2006. Isolinhas pontilhadas (contínuas) indicam valores negativos (positivos). A escala de cores abaixo da figura indica a intensidade em mm/mês. 


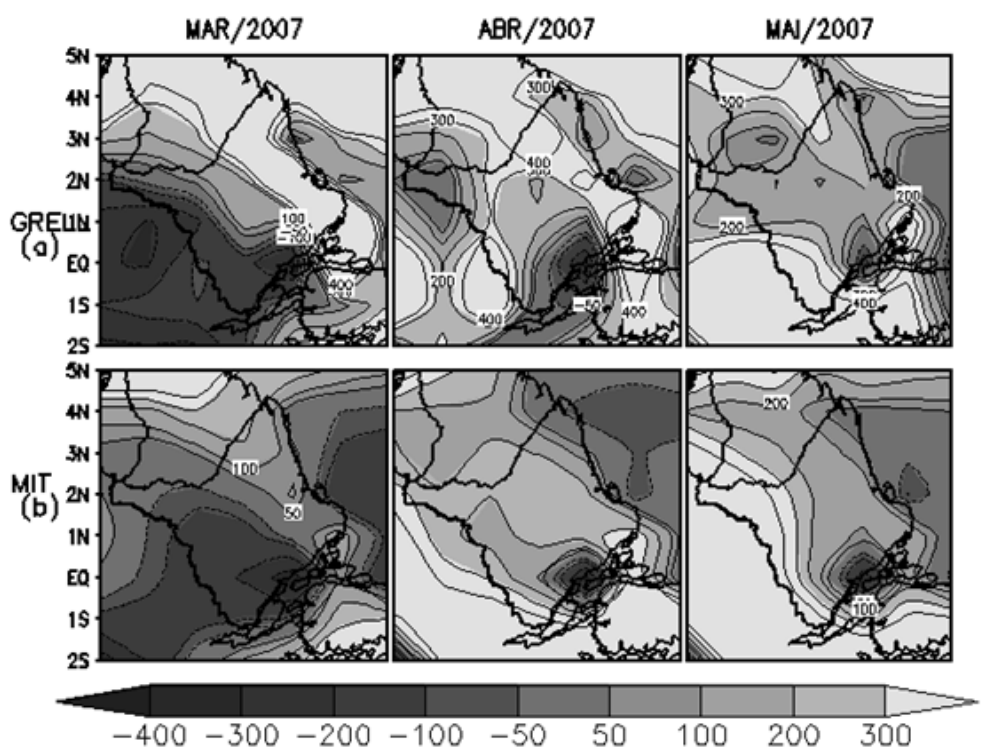

Figura 5 - (a) Viés usando o esquema de Grell, (b) Viés usando o esquema de MIT para os meses de março, abril e maio do ano de 2007. Isolinhas pontilhadas (contínuas) indicam valores negativos (positivos). A escala de cores abaixo da figura indica a intensidade em mm/mês.

Os diferentes resultados na análise quantitativa (subestimativa e superestimativa)em diferentes áreas sobre o Estado nas simulações com o RegCM3, pode estar associado as diferentes parametrizações (Grell e MIT) de convecção utilizadas, ou também, efeito da topografia (Steiner et al. 2009), além, de possíveis efeitos da resolução utilizada na simulação. Pois simulação de precipitação em modelos regionais é sensível ao tipo de resolução utilizada. (Giorgi e Marinucci (1996); Mass et al.(2002); Tanajura et al.(2003).

\section{CONCLUSÕES}

As análises mostraram uma avaliação da previsão sazonal do Modelo RegCM3 em dois eventos extremos de precipitação previamente selecionados pelo método dos quantis, para os anos 2006 e 2007. Na análise foi utilizada a resolução horizontal de $1^{\circ} \times 1^{\circ} \mathrm{Km}$ e validado com $30 \times 15$ pontos de latitude e longitude para a região do Estado do Amapá do CPC. Os resultados apresentados foram referidos a comparação entre precipitação sazonal simulada e observada para o trimestre (MAM).

De maneira geral, os resultados mostraram que a utilização da técnica de redução de escala de previsão da precipitação sazonal apresentou um bom desempenho em simular a variabilidade da chuva em escala regional. Contudo, ficaram evidentes desvios na quantidade e na posição de alguns máximos, quando comparados com o observado. Em termos qualitativos (comparação do observado com o simulado), verificou-se uma superestimativa em praticamente todos os trimestres analisados nos dois anos extremos de precipitação.
Por outro lado, em termos quantitativos (viés úmido e seco), o viés úmido foi predominante em toda faixa litorânea e o viés seco predominante nas áreas sul-sudeste, oeste e centro do Estado, com destaque para região sudeste (Macapá e Santana), que mostrou persistência de viés seco em todo o período estudado. As áreas com valores próximos do observado não foram relevantes nos resultados.

Apesar dos erros sistemáticos do RegCM3 nos dois esquemas de precipitação convectiva utilizados, o esquema de MIT se mostrou razoavelmente melhor que o Grell, em todas as simulações dos meses do período estudado(Souza et al., 2009; Da Silva et al., 2010).

Para o Estado do Amapá ainda existem poucos estudos na literatura de meteorologia com relação ao assunto abordado nesta pesquisa, para se fazer comparações entre os resultados aqui apresentados. Outros testes estão sendo feito com o RegCM3 com uma maior resolução e calibrando no aspecto do esquema de superfície-atmosfera (BATS). Estes procedimentos visam um melhor desempenho do modelo em simular a precipitação sobre o Estado e sua relação entre os processos dinâmicos da atmosfera segundo modificações do uso da terra, em especial a interação vegetação-atmosfera.

\section{AGRADECIMENTOS}

Ao CNPq, pela Concessão de bolsa de doutorado, ao Programa de Pós-Graduação em Biodiversidade Tropical (PPGBIO/UNIFAP), ao Núcleo de Hidrometeorologia e Energia Renováveis (NHMET/IEPA), ao projeto REMETAP-Finep e ao RPCH. 


\section{REFERÊNCIAS BIBLIOGRÁFICAS}

ALVES, J. M. B. et al. Um estudo inter-comparativo de previsão sazonal estatística-dinâmica de precipitação no nordeste do Brasil. Revista Brasileira de Meteorologia, v.22, n.3, p.354-372, 2007.

ALVES, L. M.; CHOU, S. C.; MARENGO, J. A. Avaliação das previsões de chuvas sazonais do modelo ETA climático sobre o Brasil. In: XIII Congresso Brasileiro de Meteorologia, 29/08 a 03/09/2004, Fortaleza-CE. Anais... Fortaleza-CE: SBMET, 2004.

CUADRA, S. V.; ROCHA, R. P. Simulação numérica do clima de verão sobre o Brasil e sua variabilidade. Revista Brasileira de Meteorologia, v.21, n.2, p.271-282, 2006.

Da SILVA, D. F. et al. Observações e simulações de precipitação com o RegCM3 no leste da Amazônia, nos anos de 1997 a 2008. In: XVI CONGRESSO BRASILEIRO DE Meteorologia, Belém-PA. Anais...Belém-PA: SBMET, 2010.

DICKINSON, R. E.; HENDERSON-SELLERS, A.; KENNEDY, P. J. Biosphere-atmosphere transfer scheme (BATS) version $1 \mathrm{E}$ as coupled to the NCAR Community Climate Model. Boulder, Colorado: Tech Note NCAR/TN-387, 72pp, 1993.

DRUYAN, L. M.; FULAKEZA, M.; LONERGAN, P. Dynamic downscaling of seasonal climate predictions over Brazil. Journal of Climate, v.15, p.3411-3426, 2002.

ELGUINDI, N. et al. RegCM version 3.0 user's guide. Trieste: PWCG Abdus Salam ICTP, 48 pp, 2004.

EMANUEL, K. A. A scheme for representing cumulus convection in large-scale models. Journal of Atmospheric Sciences, v.48, p.2313-2335, 1991.

FERNANDEZ, J. P. R.; FRANCHITO, S. H.; RAO, V. B. Simulation of the summer circulation over South America by two regional climate models. Part I: Mean climatology. Theorical and Applied Climatology, v.86, p.247-260, 2006.

FIGUEROA, S. N.; NOBRE, C. A. Precipitation distribution over central and western tropical South America. Climanálise, 5(6): p.36-45, 1990.

FRIAS, M. D. et al. Operational predictability of monthly average maximum temperature over the Iberiam Peninsula using DEMETER simulation and downscaling. Tellus 57A: p.448-463, 2005.

GIORGI, F.; MARINUCCI, M. An investigation of the sensitivity of simulate precipitation to model resolution and its implication for climate studies. Monthly Weather Review, v. 124, n.34, p.148-146, 1996.

GIORGI, F.; MEARNS, L. O. Approaches to the simulation of regional climate change: A review. Journal of Geophysical Research, Washington, v. 29, p.191-216, 1991.
GIORGI, F.; MEARNS, L.O. Introduction to especial section: Regional climate modeling revisited. Journal of Geophysical Research, v.104, n.D6, p.6335-6352, 1999.

GRELL, G. A. Prognostic evaluation of assumptions used by cumulus parameterization. Monthly Weather Review, Boston, v.121, p.764-787, 1993.

HOLTSLAG, A.; BRUIJN, E. I. F.; PAN, H. L. A high resolution air mass transformation model for short-range weather forecasting. Monthly Weather Review, Boston, v.118, p.1561-1575, 1990.

KALNAY, E. et al. NCEP/NCAR 40-year Reanalysis project. Bulletin of the American Meteorological Society, v.77, p.437-471, 1996.

KIEHL, J. T. et al. Description of the NCAR Community Climate Model (CCM3). Boulder, Colorado: Technical Note, NCAR/TN- 420+STR, 152 pp, 1996.

LOPES, M. N. G.; Aspectos regionais da variabilidade de precipitação no Estado do Pará: estudo observacional e modelagem climática em alta resolução. Dissertação de Mestrado, Universidade Federal do Pará/ Faculdade de Meteorologia/ Programa de Pós-Graduação em Ciências Ambientais. Belém-PA. 80p, 2009.

MASS, C. F. et al. Does increasing horizontal resolution produce more skillful forecasts. American Meteorological Society. v. 83, n.3. p.407-430. 2002.

PAL, J. S. et al. The ICTP RegCM3 and RegCNET: regional climate modeling for the developing World. Bulletin of the American Meteorological Society, v.1, p.205-212, 2007.

QIAN, J. H.; ANJI, S.; STEPHEN, Z. Reinitialized versus continuous simulation for regional climate downscaling. Monthly Weather Review, v.131, p.2857-2874, 2003.

REYNOLDS, R. W. et al. An improved in situ and satellite SST analysis for climate. Journal ofClimate, v.15, p.1609$1625,2002$.

SETH, A.; ROJAS, M. Simulation and sensitivity in a nested modeling system for South American. Part I: Reanalyses boundary forcing. Jornal of Climate, v.16, p.2437-2453, 2003.

SILVA, V. B. S. et al.Ana improved gridded historical daily precipitation analysis for Brazil. Journal of Hydrometeorology, 8:847-861, 2007.

SOUZA, E. B. et al. Precipitação sazonal sobre a Amazônia oriental no período chuvoso: Observações e simulações regionais com o RegCM3. Revista Brasileira de Meteorologia, v.24, n.2, p.111-124, 2009.

SOUZA, E. B.; KAYANO, M. T.; AMBRIZZI, T. The regional precipitation over the eastern Amazon/northeast Brazil modulated by tropical pacific and Atlantic SST anomalies on weekly timescale. Revista Brasileira de Meteorologia, v.19, n.3, p.325-336, 2004. 
SOUZA, E. B.; ROCHA, E. J. P. Diurnal variation of rainfall in Bragança-PA (eastern Amazon) during rainy season: mean characteristic and extremes events. Revista Brasileira de Meteorologia, v.21, n.3a, p.142-152, 2006.

STEINER, A. L. et al. Land surface coupling in regional climate simulations of the West African monsoon. Climate Dynamic, 33:869-892,2009.

TANAJURA, C. A. S.; CÁRDENAS, W. C.; BRANCO, R. A. The influence of the domain and resolution on the ETA model simulation of a cold front passage over southeast Brazil. Revista Brasileira de Meteorologia, v.18, n.1, p.21-42, 2003.
XAVIER, T. DE Mª. B. S.; XAVIER, A. F. S. Classificação e monitoramento de períodos secos ou chuvosos e cálculo de índices pluviométricos para a região nordeste do Brasil. Revista Brasileira de Engenharia, v.5, n.2, 1987. 